\title{
Impact of beating heart left atrial ablation on left-sided heart mechanics
}

\author{
Takeyoshi Ota, MD, PhD ${ }^{a}$, David Schwartzman, MD ${ }^{\mathrm{b}}$, David Francischelli, MS ${ }^{\mathrm{c}}$, Douglas A. Hettrick, $\mathrm{PhD}^{\mathrm{c}}$, and \\ Marco A. Zenati, MD
}

From the Division of Cardiac Surgery and Cardiovascular Institute, ${ }^{\mathrm{b}}$ University of Pittsburgh, Pittsburgh, Pa; and Medtronic, Inc, ${ }^{\mathrm{c}}$ Minneapolis, Minn.

David Francischelli reports that he is an employee of Medtronic, the manufacturer of the ablation tool used in this study, an equity owner of Medtronic, and the inventor of patents related to the ablation device with rights assigned to Medtronic. Douglas Hettrick is an employee of Medtronic. David Schwartzman reports consulting fees and grant support from Medtronic. Marco Zenati reports grant support from Medtronic. This study was supported in part by Medtronic.

Received for publication Dec 4, 2006; revisions received March 29, 2007; accepted for publication April 9, 2007.

Address for reprints: Marco A. Zenati, MD, Division of Cardiac Surgery, University of Pittsburgh, 200 Lothrop St, PUH C-700, Pittsburgh, PA 15213-2582 (E-mail: zenatim@upmc.edu).

J Thorac Cardiovasc Surg 2007;134:982-8

$0022-5223 / \$ 32.00$

Copyright (๑) 2007 by The American Association for Thoracic Surgery

doi:10.1016/j.jtcvs.2007.04.063
Objective: The cut-and-sew Cox-Maze procedure is the gold standard for surgical treatment of atrial fibrillation, but it is associated with long-term impairment of left atrial mechanical function. We developed a bipolar, irrigated radiofrequency ablation device. We hypothesized that beating heart radiofrequency left atrial ablation would result in minimal acute changes in left atrial hemodynamics.

Methods: Six healthy subjects were studied. Combination pressure-conductance catheters were inserted into the left atrium and ventricle. With the use of the device, atrial ablation was performed on the beating heart without cardiopulmonary bypass, including electrical isolation of the posterior left atrium and atrial appendage myocardium. Simultaneous left-sided heart pressure-volume and intracardiac echocardiography data were acquired before ablation, after left atrial appendage ablation alone, and after all ablation (with and without appendage occlusion). The derived indices of left-sided heart mechanical function were examined.

Results: Relative to baseline, no significant diminishment in pressure-volume or intracardiac echocardiography-derived indices of global left-sided heart mechanical function were observed after ablation, with or without appendage occlusion. Mitral valve morphology and function were not significantly altered. A significant diminishment of atrial appendage systolic flow was noted after appendage ablation in association with spontaneous echocardiographic contrast in this region.

Conclusions: In this model, ablation does not seem to compromise global left-sided heart mechanical function. However, these findings mask regional diminishment in atrial appendage systolic function. This observation demonstrates that electrical isolation of the appendage should be accompanied by its occlusion or excision. Appendage occlusion after ablation does not seem to compromise left-sided heart mechanical function.

A $\mathrm{n}$ ideal treatment for atrial fibrillation (AF) should achieve both restoration and maintenance of sinus rhythm and recovery of biatrial contractile function. In addition, an integral component of any therapeutic approach for AF should be the reduction of thromboembolic risk primarily targeting the left atrial (LA) appendage. As operative atrial ablation intended to cure or prevent AF progresses toward the mainstream, questions have appropriately been raised as to the possibility that this technique may have detrimental effects. Apart from prolongation of a concomitant surgical procedure, such effects could potentially include mechanical, intrinsic cardiac neurologic, and/or humoral impairment. For example, evaluation of atrial function after cut-and-sew Maze III surgery indicated that diminishment of LA mechanical function may result from the surgical lesions and tissue excision. ${ }^{1,2}$

Unlike in cut-and-sew Maze III surgery, recent advances in ablation technology have permitted the deployment of lesions in the beating heart and with less atriotomy. ${ }^{2}$ In addition, expanding insight into the atrial electrophysiologic substrate 


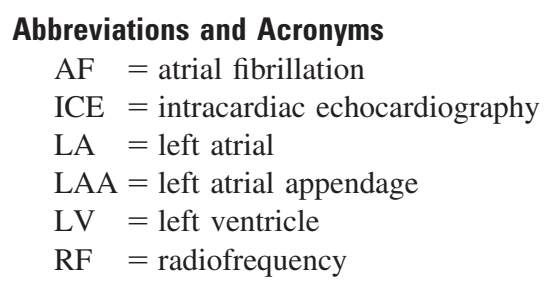

has led to a progressive reduction in ablation territory relative to that of classic Maze surgery. ${ }^{2}$ Each of these elements has the potential to influence the impact of the surgery on cardiac mechanical function.

In the present study, we sought to assess the impact of LA ablation on left-sided heart mechanical function using a porcine model in which a typical contemporary lesion set was deployed in a beating heart.

\section{Materials and Methods}

Animals

Six healthy Duroc cross female pigs (weight $36 \pm 13 \mathrm{~kg}$ ) formed the study cohort. We and others previously demonstrated that atrial chamber dimensions, blood flow velocities, and wall thicknesses are similar to those of a human adult. ${ }^{3,4}$ All animals received humane care in a facility sanctioned by the Council on Accreditation of the Association for Assessment and Accreditation of Laboratory Animal Care, in accordance with the "Guide for the Care and Use of Laboratory Animals" published by the National Institutes of Health (publication 85-23, revised 1985). The study protocol was approved by the Institutional Animal Care and Use Committee of the University of Pittsburgh.

\section{Operative Technique}

Animals were premedicated with intramuscular ketamine and inhaled isoflurane before endotracheal intubation. Once the animals were intubated, a surgical plane of anesthesia was achieved and maintained with isoflurane $1 \%$ to $5 \%$ inspired. Arterial blood pressure, blood gases, and serum electrolytes were monitored serially. Each animal was systemically anticoagulated (heparin, adjusted for body weight) for the duration of the procedure. For insertion of diagnostic catheters, endocardial access to the right atrium was gained through a right femoral venipuncture, to the LA subsequently through an atrial transseptal puncture, and to the left ventricle (LV) through a carotid arteriotomy and retrograde transaortic passage. For ablation, epicardial access to the LA was gained through median sternotomy and pericardiotomy, and endocardial access was gained through focal atriotomies (see below). Some dissection in the posterior mediastinum was necessary to create sufficient access

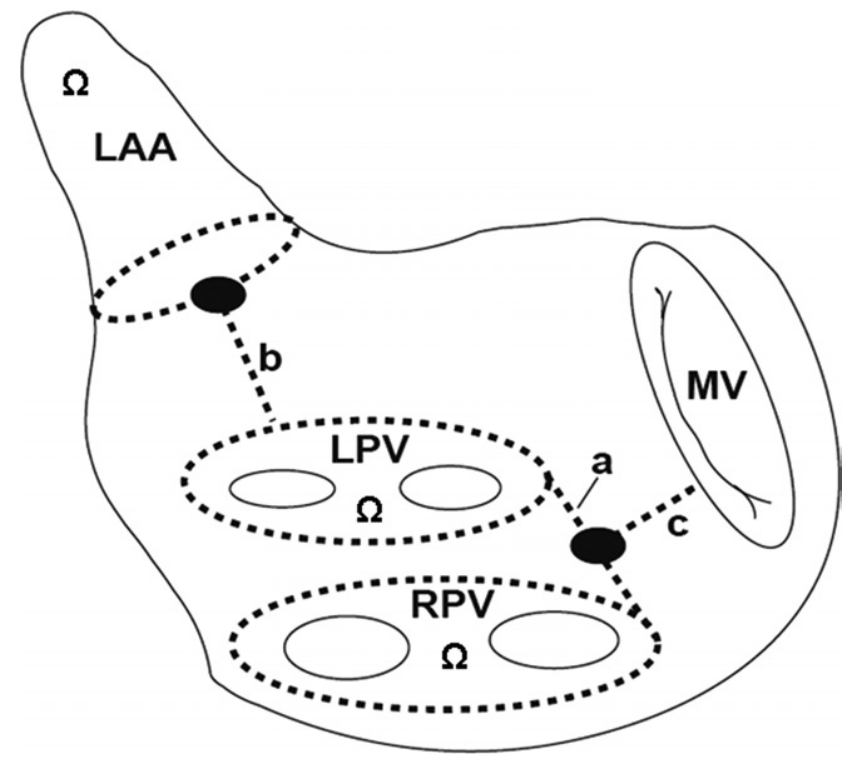

Figure 1. Ablation lesions, encircling lesions (dashed circles), and linear lesions (dashed lines, A-C). Approximate atriotomy sites $(\bullet)$ required for access of 1 of the jaws of the ablation device during deployment of linear lesions (see text). Approximate sites $(\Omega)$ of pacing to assess for exit conduction block after encircling lesions (see text). $L A A$, Left atrial appendage; $L P V$, left pulmonary veins; $R P V$, right pulmonary veins; $M V$, mitral valve.

for the ablation device; this required approximately 15 minutes per animal. At the completion of the experiment, each animal was euthanized while under deep anesthesia using an injection of potassium chloride.

\section{Ablation}

Device. Ablation was performed using a commercial bipolar device (Cardioblate BP2, Medtronic Inc, Minneapolis, Minn). ${ }^{5}$ The device was composed of 2 stainless steel electrodes, arrayed as cathode and anode on opposing jaws of a clamp-morphology skeleton, and embedded in a porous polymer through which room-temperature normal saline was irrigated at a rate of $4 \mathrm{~mL} / \mathrm{min}$. The device was coupled to a commercial radiofrequency (RF) energy generator (Cardioblate, Medtronic Inc) from which real-time (updated every $200 \mathrm{~ms}$ ) impedance, power, lesion duration, and cumulative delivered energy data were obtained. The generator used a proprietary algorithm that titrated power, assessed lesion transmurality based on changes in impedance, and signaled the user when the lesion was completely transmural.

Lesion deployment and assessment. In each animal, the same lesion set was deployed in the beating heart without cardiopulmonary bypass (Figure 1). Encircling lesions (appendage and pulmonary veins) were applied 

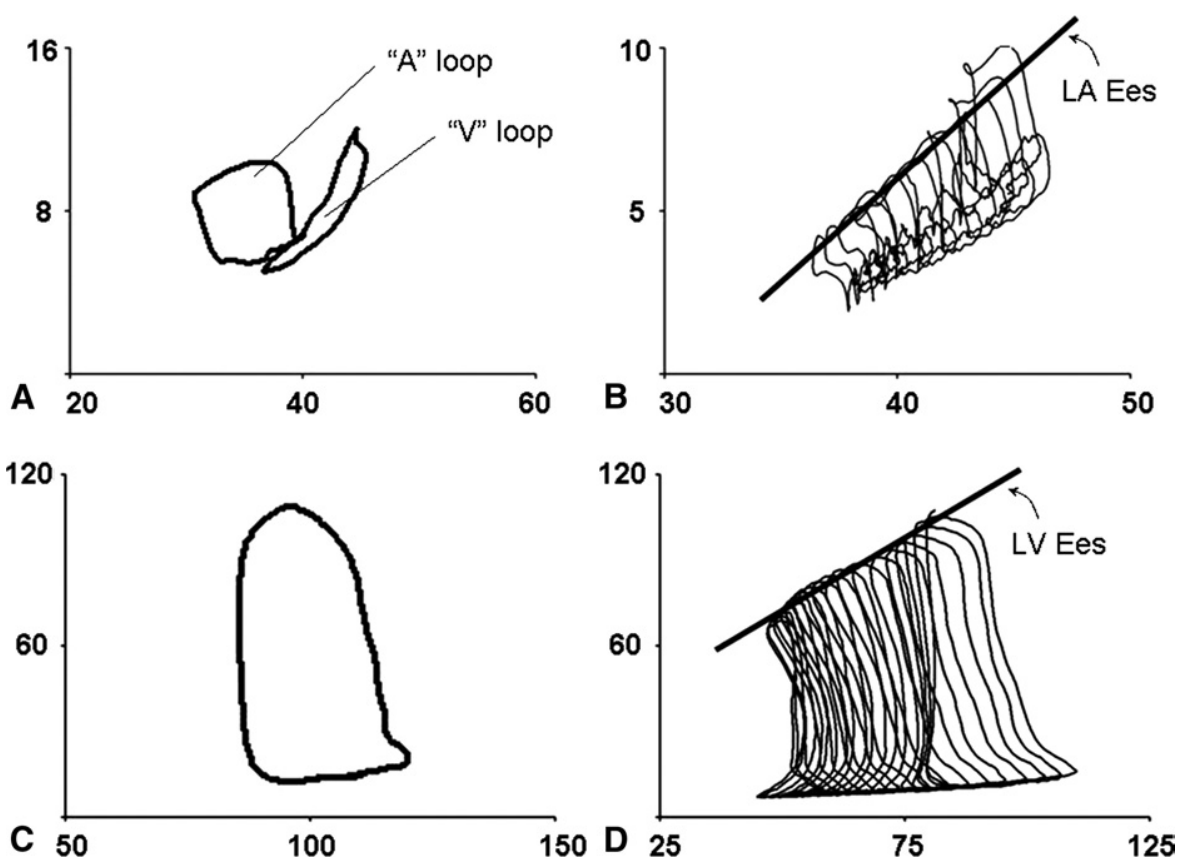

Figure 2. Typical examples of passive (no perturbation; left) and active (inferior caval constriction; right) LA (top row) and LV (bottom row) pressure (Y axis, in millimeters of mercury)-volume ( $X$ axis, in milliliters) relationships. The LA relationships demonstrate typical bifid morphology containing "A loop" (demarcating atrial booster pump function) and "V loop" (demarcating passive changes in LA pressure and volume generated by LV activity). End-systolic elastance was measured for each chamber as the slope of the line (demonstrated) comprised by connecting end-systolic points of the series of pressure-volume relationships generated during inferior caval constriction. ${ }^{8} L A$, Left atrial; Ees, end-systolic elastance. by clamping the epicardium only. Linear lesions were applied with jaws located contiguously, 1 endocardial and 1 epicardial, after access was gained through focal atriotomies (Figure 1): lesions B (except 1 animal; see below) and $\mathrm{C}$ were each performed with a single $\mathrm{RF}$ application; because of limited access, lesion A was performed in 2 adjacent RF applications, during which the jaws of the device were oriented in opposite directions through a purse-string access. Note that the path of lesion $\mathrm{C}$ was chosen to avoid the portion of the circumflex coronary proximal to obtuse marginal branches; in all pigs, the coronary artery distribution was right coronary artery dominant. The left atrial appendage (LAA) was not excised. The order of lesion application was the same in each animal, with the LAA encircling lesion first, followed by left then right pulmonary vein encircling lesions, and then linear lesions B, A, and C.

Immediately after lesion deployment, assessment of electrical conduction across the encircling lesions was performed by unipolar (7F, 2-mm length passive fixation cathode; noncardiac intrathoracic anode) epicardial pacing of myocardium subtended by each of the encircling lesions after each RF application at a cycle length $100 \mathrm{~ms}$ less than sinus and an output of $20 \mathrm{~mA}$ (Figure 1). Conduction (as defined by acceleration of the ventricular cycle length to that of pacing) from each of these same territories was demonstrated at baseline. Exit conduction block was defined by absence of this response. No assessment of conduction across the linear lesions was performed.
Evaluation of Left-sided Heart Mechanics

The following 2 techniques were used:

1. Pressure-volume analysis: The utility of using conductance to assess time-varying changes in global cardiac chamber volumes has been described in detail. $^{6}$ Before ablation, 2 commercial duodecapolar conductance catheters, each incorporating a pressure sensor (CD Leycom, Inc, Zoetermeer, The Netherlands), were inserted into the heart: 1 into the $\mathrm{LV}$ and 1 into the LA. Each catheter insertion was guided by intracardiac echocardiography (ICE; see below) to ensure that appropriate positioning (defined by: i. all electrodes located in the chamber and ii. catheters location approximately midcavity, with no endocardial contact) was achieved. Subsequently, in each animal ICE was used to monitor catheter positions to ensure positional constancy throughout the experimental protocol (see below). Conductance data from each catheter were collected simultaneously, as was pressure, with 2 commercial acquisition systems (CFL-512, CD Leycom, Inc) using unique stimulation frequencies to avoid interference. Data from each system were analyzed using investigational software (Conduct NT, CD Leycom, Inc). Blood resistivity was measured at baseline and at the end of the experimental protocol. Data were obtained in stable state and during transient manual constriction of the inferior caval vein using a surgical tape; the latter was used for calculations of LA and LV end-systolic elastance, which assumed a linear fit to the progres- 
TABLE 1. Data derived from pressure-volume analysis

\begin{tabular}{|c|c|c|c|c|}
\hline & Baseline & $\begin{array}{l}\text { After appendage } \\
\text { ablation only }\end{array}$ & $\begin{array}{l}\text { After all ablation without } \\
\text { appendage occlusion }\end{array}$ & $\begin{array}{l}\text { After all ablation with } \\
\text { appendage occlusion }\end{array}$ \\
\hline $\mathrm{LV}+\mathrm{dP} / \mathrm{dtmax}(\mathrm{mm} \mathrm{Hg} / \mathrm{sec})$ & $967 \pm 334$ & $975 \pm 304$ & $878 \pm 193$ & $848 \pm 122$ \\
\hline LV stroke work (mL/mm Hg) & $1317 \pm 896$ & $1115 \pm 780$ & $1336 \pm 782$ & $1403 \pm 852$ \\
\hline $\begin{array}{l}\text { LV end-systolic elastance } \\
\text { slope }^{*}(\mathrm{~mm} \mathrm{Hg} / \mathrm{mL})\end{array}$ & $4.9 \pm 3.4$ & $3.8 \pm 2.5$ & $3.0 \pm 2.1$ & $3.2 \pm 3.5$ \\
\hline LV Taut(ms) & $28 \pm 6$ & $28 \pm 8$ & $29 \pm 7$ & $30 \pm 8$ \\
\hline LA mean pressure $(\mathrm{mm} \mathrm{Hg})$ & $5.9 \pm 4.1$ & $6.2 \pm 4.0$ & $5.8 \pm 3.7$ & $7.1 \pm 4.3$ \\
\hline LA A loop area $(\mathrm{mL} / \mathrm{mm} \mathrm{Hg})$ & $28 \pm 22$ & $31 \pm 16$ & $15 \pm 10$ & $38 \pm 43$ \\
\hline LA V loop area§(mL/mm Hg) & $25 \pm 32$ & $38 \pm 52$ & $19 \pm 9$ & $34 \pm 17$ \\
\hline $\begin{array}{l}\text { LA end-systolic elastance slope } \\
\text { (mm Hg/mL) }\end{array}$ & $0.61 \pm 0.25$ & $0.38 \pm 0.17$ & $0.59 \pm 0.26$ & $0.52 \pm 0.22$ \\
\hline $\begin{array}{l}\text { LA end-diastolic elastance slope\| } \\
\text { (mm Hg/mL) }\end{array}$ & $0.31 \pm 0.25$ & $0.49 \pm 0.51$ & $0.41 \pm 0.13$ & $0.50 \pm 0.23$ \\
\hline $\begin{array}{l}\text { Maximum LA elastance: LV passive } \\
\text { stiffness ratiol }\end{array}$ & $0.99 \pm 0.94$ & $0.75 \pm 0.92$ & $0.84 \pm 0.64$ & $1.08 \pm 0.82$ \\
\hline
\end{tabular}

$L A$, Left atrium; $L V$, left ventricle. *A “load-independent" index of global $\mathrm{LV}^{19}$ or $\mathrm{LA}^{8}$ contractility. †Time constant of $\mathrm{LV}$ isovolumic relaxation. ${ }^{20} \neq I n d i c e s$ of active (eg, delivery of energy derived from atrial myocyte contraction) work performed by the LA. ${ }^{8}$ §Indices of passive (eg, delivery of stored energy derived from LV contraction) work performed by the LA. ${ }^{8}$ "A direct measure of passive LA stiffness. ${ }^{8}$ ๆAn index of mechanical "coupling" between the LA and LV that quantifies the relation between the contractile state of the LA and the passive LV properties resisting its ejection. ${ }^{21}$

sion of data (Figure 2). Data derived in the stable state were an average of 10 consecutive cardiac cycles. Table 1 lists the indices of mechanical function derived from pressure-volume data.

2. ICE: A commercial system was used (AcuNAV, Siemens Medical Solutions, Malverne, Pa) that includes a $10 \mathrm{~F}$ deflectable catheter incorporating a phasedarray transducer with programmable operating frequency. ${ }^{7}$ In the present study, this catheter was placed as necessary into the right atrium and caval veins, where it was rotated and deflected to achieve the desired imaging planes. The imaging frequency ranged between 5 and $7.5 \mathrm{mHz}$. The system is equipped with 2-dimensional imaging and pulsed, continuous-wave, and color Doppler. In addition to guiding placement and monitoring stability of pressure-volume catheters (above), ICE was used to serially assay atrial mechanical function using the indices listed in Table $2,{ }^{8-11}$ and to detect the presence of intracavitary left-sided heart thrombi.

\section{Pathologic Analysis}

Hearts were first examined in situ to assess for damage to noncardiac structures contiguous to the lesions. The heart and lungs were then removed en bloc. Gross visual inspection was performed from epicardial and endocardial van-

TABLE 2. Data derived from intracardiac echocardiographic analysis

\begin{tabular}{|c|c|c|c|c|}
\hline & Baseline & $\begin{array}{l}\text { After appendage } \\
\text { ablation only }\end{array}$ & $\begin{array}{l}\text { After all ablation without } \\
\text { appendage occlusion }\end{array}$ & $\begin{array}{l}\text { After all ablation with } \\
\text { appendage occlusion }\end{array}$ \\
\hline $\begin{array}{l}\text { Peak antegrade left pulmonary } \\
\text { vein flow velocity }(\mathrm{m} / \mathrm{sec})\end{array}$ & $1.2 \pm 0.4$ & $1.4 \pm 0.5$ & $1.1 \pm 0.5$ & $1.6 \pm 1.1$ \\
\hline $\begin{array}{l}\text { Peak retrograde left pulmonary } \\
\text { vein flow velocity }(\mathrm{m} / \mathrm{sec})\end{array}$ & $0.2 \pm 0.2$ & $0.1 \pm 0.1$ & $0.1 \pm 0.3$ & $0.1 \pm 0.2$ \\
\hline $\begin{array}{l}\text { Peak antegrade right pulmonary } \\
\text { vein flow velocity }(\mathrm{m} / \mathrm{sec})\end{array}$ & $1.1 \pm 0.2$ & $0.9 \pm 0.4$ & $1.3 \pm 0.5$ & $1.3 \pm 0.7$ \\
\hline $\begin{array}{l}\text { Peak retrograde right pulmonary } \\
\text { vein flow velocity }(\mathrm{m} / \mathrm{sec})\end{array}$ & $0.1 \pm 0.2$ & $0.3 \pm 0.4$ & $0.1 \pm 0.1$ & $0.1 \pm 0.5$ \\
\hline $\begin{array}{l}\text { Peak antegrade transmitral flow } \\
\text { velocity }(\mathrm{m} / \mathrm{sec})\end{array}$ & $2.6 \pm 0.5$ & $2.8 \pm 0.9$ & $2.4 \pm 0.8$ & $2.2 \pm 1.2$ \\
\hline Mitral regurgitant fraction $(\%)$ & $3 \pm 1$ & $1 \pm 1$ & $6 \pm 4$ & $7 \pm 4$ \\
\hline $\begin{array}{l}\text { Peak atrial appendage contraction } \\
\text { wave velocity }(\mathrm{m} / \mathrm{sec})\end{array}$ & $0.5 \pm 0.2$ & $0.1 \pm 0.2^{*}$ & $0.1 \pm 0.1^{*}$ & 0 \\
\hline
\end{tabular}

${ }^{*} P<.05$ versus baseline. 
TABLE 3. Ablation and pathologic data

\begin{tabular}{lcccc}
\hline Lesion & $\begin{array}{c}\text { Total energy delivered } \\
\text { in Joules }\end{array}$ & $\begin{array}{c}\text { Duration of application } \\
\text { in seconds }\end{array}$ & $\begin{array}{c}\text { Lesion length in } \\
\text { centimeters }\end{array}$ & $\begin{array}{c}\text { Lesion width in } \\
\text { millimeters }\end{array}$ \\
\hline LAA encircling & $519 \pm 117$ & $18 \pm 5$ & $6.1 \pm 1.4$ & $4.1 \pm 1.1$ \\
LPV encircling & $424 \pm 40$ & $16 \pm 2$ & $4.1 \pm 0.9$ & $1.9 \pm 1.4$ \\
RPV encircling & $474 \pm 23$ & $17 \pm 2$ & $3.7 \pm 1.3$ & $2.9 \pm 0.9$ \\
Linear "a" & $326 \pm 108$ & $18 \pm 5$ & $2.3 \pm 0.6$ & $2.4 \pm 1.0$ \\
Linear "b" & $469 \pm 212$ & $17 \pm 7$ & $3.1 \pm 1.1$ & $2.0 \pm 0.7$ \\
Linear "c" & $390 \pm 81$ & $14 \pm 3$ & $1.8 \pm 0.8$ & $2.2 \pm 1.3$ \\
\hline
\end{tabular}

$L A A$, Left atrial appendage; $L P V$, left pulmonary veins; $R P V$, right pulmonary veins. Linear "a, b, and c" are indicated in Figure 1.

tages. A particular interest was to assess the damage to the mitral valve apparatus and the appearance of the ablation lesion in coronary sinus tissue. Ablation lesions were examined to assess for evidence of charring or barotrauma. ${ }^{4}$ The heart was then immersed in $1 \%$ triphenyl tetrazolium chloride for 60 minutes, after which atrial wall thickness and lesion dimensions (length, depth, and endocardial/epicardial surface thicknesses) were measured. ${ }^{4}$ Histologic lesion transmurality was defined by exclusion of tetrazolium stain spanning from endocardium to epicardium.

\section{Experimental Protocol}

Pressure-volume and ICE data were acquired at each of 4 phases of the procedure: (1) baseline: after thoracotomy and posterior mediastinal dissection, immediately before ablation lesion deployment; (2) after LAA ablation only; (3) after all ablation; (4) after all ablation, with the appendage completely occluded using a plastic clamp, confirmed by ICE. Each data set was acquired with the pericardial and thoracotomy edges reapposed and the lungs at functional residual capacity. Data sets 2, 3, and 4 were each acquired after 5 to 10 minutes of post-intervention steady state.

\section{Analytic Methods}

Data are reported as mean \pm standard deviation, unless otherwise specified. Within-animal differences in pressurevolume and ICE indices between experimental stages were assessed for statistical significance using a Friedman repeated-measures analysis of variance, followed by a StudentNewman-Keuls multiple comparisons test.

\section{Results}

\section{Operative Notes}

The cardiac rhythm was sinus throughout the procedure. The baseline sinus cycle length $(710 \pm 65 \mathrm{~ms})$ did not change significantly during other phases of the experimental protocol. All animals were stable throughout the procedure, including systemic blood pressure, gas exchange, acid-base status, electrolytes, and blood resistivity. During device manipulation and ablation lesion application, we commonly performed brief manual compression of the inferior caval vein to enhance LA flaccidity, which facilitated ablation electrode access/fixation and minimized blood loss. In 1 animal, territory length necessitated that lesion B was performed with 2 contiguous applications; in all other animals a single application was sufficient. In 1 animal, 2 RF applications were necessary to achieve exit conduction block of the LAA: Visual inspection after the first lesion revealed that the jaws of the device had been malpositioned, excluding a portion of tissue that presumably was the conduit through which there was persistent conduction. After an additional application using a more appropriate device position, exit conduction block was demonstrated. In all animals, exit conduction block was noted after single RF applications in both right and left pulmonary vein regions. The entire experimental protocol was completed in all animals in less than 30 minutes. Ablation data are summarized in Table 3. No significant interlesional differences in ablation energy requirement or duration were observed.

\section{Assessment of Impact of Ablation on Mechanics}

Pressure-volume. Relative to baseline, no significant difference was noted in any of the derived indices after appendage ablation alone or after all ablation with or without appendage occlusion (Table 1).

Intracardiac echocardiograph. Ablation did not alter the morphology of the mitral leaflet. At no time was there evidence of intracavitary thrombus. After appendage ablation, spontaneous echo contrast was noted in the distal appendage region, suggestive of flow stasis. Relative to baseline, no significant differences were noted in the transvenous or transmitral velocities after ablation. Similarly, the mitral regurgitant fraction was not significantly altered. There was, however, a significant decrease in the peak atrial appendage contraction wave velocity after ablation of the appendage (Table 2).

\section{Pathologic Analysis}

At postmortem, there was no evidence of collateral damage to cardiac or noncardiac structures. None of the lesions evidenced thrombus or barotrauma (Figure 3). Lesion C 


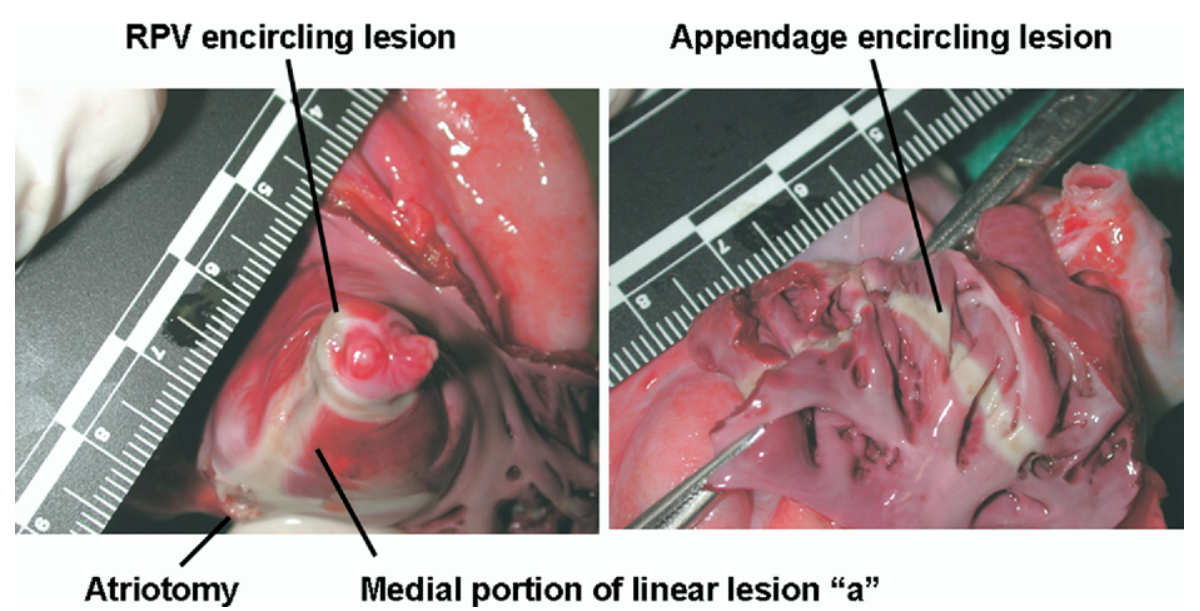

Figure 3. Photographs taken from endocardial vantage demonstrating typical lesion morphologies (post-tetrazolium staining) in territories of smooth (left) and trabeculated (right) endocardial topography. RPV, Right pulmonary vein.

abutted the posterior mitral leaflet, but there was no perceptible damage to the leaflet or contiguous chordal apparatus. Lesion $\mathrm{C}$ also produced a transmural encircling lesion of the included coronary sinus musculature.

Ablation lesions were uniform and transmural throughout their course; the mean mural lesion thickness was $2.3 \pm$ $0.8 \mathrm{~mm}$ (range 1-6 mm) in regions of smooth endocardial topography (pulmonary vein encircling lesions and linear lesions $\mathrm{A}$ and $\mathrm{C}$ ) and $3.1 \pm 1.9 \mathrm{~mm}$ (range $1-8 \mathrm{~mm}$ ) in regions of trabeculated endocardial topography (appendage encircling lesion and linear lesion B). Individual lesion dimensions are summarized in Table 3.

\section{Discussion}

We used a method for LA ablation in a beating heart without the need for cardiopulmonary bypass. Ablation was performed quickly and without complications, yielding ablation/isolation of a relatively large territory of myocardium. ${ }^{4,5}$ To assess the impact of the procedure on left-sided heart mechanical function, we used pressure-volume and echocardiographic analytic techniques in tandem. To our knowledge, this is the first study to use pressure-volume analysis for this purpose; in addition, the simultaneous acquisition of LA and LV data, which permits assay of individual interchamber coupling, is also novel. Our data confirm our prior observation that this procedure did not seem to adversely affect LA booster pump function. ${ }^{4,5}$ The data also extend these observations, demonstrating that ablation did not seem to have an adverse affect on intrinsic LA contractility (elastance), passive LA mechanical properties (reservoir, conduit), LV systolic and diastolic mechanics, or the vigor with which the LA and LV were mechanically coupled. $^{8}$

As we previously reported, ${ }^{4}$ the lack of adverse impact in global indices of left-sided heart mechanical function demonstrated here masked a potentially important regional diminishment in LAA function. Given that it was also ob- served after appendage ablation but before ablation in the remainder of the atrium, it is likely that this was primarily the result of electrical isolation of appendage tissue, which eliminated its electromechanical activation. On the basis of the echocardiographic features of the appendage after ablation, including diminished flow and spontaneous echo contrast, we conclude as before that electrical isolation of the appendage without mechanical isolation would be unwise. ${ }^{4}$ Our data suggest that mechanical isolation would not adversely affect global left-sided heart mechanical function.

We note several important limitations to these data. First, the experiment was performed in animals with healthy hearts and ablation was not performed concomitantly with other cardiac surgery. Abnormal cardiac structure and concomitant surgery are prominent in the human Maze experience. It is likely that preexisting atrial/ventricular dysfunction with or without concomitant surgery would have yielded a different result. Also, as is true for any animal experiment, the applicability of these findings to humans is unaddressed. Second, assessment of LA mechanics using a conductance technique has not been well studied. Of particular concern is that spatially irregular, small-volume regions such as the appendage may not be represented in the conductance field or aggregate data. This concern is supported by the significant changes in LAA function demonstrated by echocardiography. Third, our preparation involved median sternotomy and pericardiotomy, which could have potentially masked adverse mechanical impact that would have been apparent in a closed-chest preparation. Fourth, our data are acute only and therefore do not address the possibility of a delayed adverse impact on cardiac mechanics. Included in this consideration would be the possibility that resulting intrinsic cardiac neurologic and/or humeral perturbations could adversely affect mechanics. ${ }^{12}$ In regard to this, in a chronic study using a similar animal model, Melby and colleagues ${ }^{13}$ demonstrated a significant diminishment in LA motility at 1 month post ablation. 
Similarly, it is important to note that acute lesion histology may not accurately portray chronic lesion histology; this could include an element of "healing." Fifth, these data are limited to the ablation paradigm (lesion pattern and ablation tool) described here. Although we believe that an important element contributing to the lack of adverse mechanical impact was avoidance of damage to Bachmann's Bundle, ${ }^{4}$ our data do not address this issue or the impact of other lesion paradigms (left and/or right atrial), regardless of whether they involve ablation in the region of Bachmann's Bundle. Sixth, our findings suggesting that appendage occlusion would not significantly alter left-sided heart mechanics are in contrast with the findings of previous studies in which significant alterations were noted. ${ }^{14-17}$ Possible explanations for this variance include the model, the thoracotomy, the fact that the appendage was clamped but not excised, and the fact that the assessment was made only after all ablation lesions were deployed. Seventh, no effort was made to assess the impact of the ablation procedure on AF inducibility/sustainability. Our goal was to assess the impact of ablation on left-sided heart mechanical function. Although we are dubious as to the clinical relevance of arrhythmia suppression assessments in animal models, we do note the high rate of AF suppression reported using a lesion set similar to ours in a canine model. ${ }^{18}$ Eighth, we note that our conclusions are derived from a small cohort and await prospective confirmation. Finally, it is important to emphasize that although the tool used here is commercially available, its use in human AF ablation is currently off-label (a clinical study intended to change the labeling is ongoing). In addition, a specific contraindication is included in the labeling for use in a "blood pool," as was the case for the lesions that necessitated atriotomy. As an additional note of caution, we remind readers that although we did not observe functional damage to the mitral apparatus after annular ablation, it has been reported elsewhere. ${ }^{13}$

\section{References}

1. Feinberg M, Waggoner A, Kater K, Cox J, Lindsay B, Perez J. Restoration of atrial function after the maze procedure for patients with atrial fibrillation: assessment by Doppler echocardiography. Circulation. 1994;90:285-92.

2. Melby S, Zierer A, Bailey M, Cox J, Lawton J, Munfakh N, et al. A new era in the surgical treatment of atrial fibrillation: the impact of ablation technology and lesion set on procedural efficacy. Ann Surg. 2006;244:583-92.
3. Ren J, Schwartzman D, Callans D, Marchlinski F. Intracardiac echocardiography $(9 \mathrm{MHz})$ in humans: methods, imaging views, and clinical utility. Ultrasound Med Biol. 1999;25:1077-86.

4. Schwartzman D, Bonanomi G, Zenati M. Epicardium-based left atrial ablation: impact on electromechanical properties. J Cardiovasc Electrophysiol. 2003;14:1087-92.

5. Bonanomi G, Schwartzman D, Francischelli D, Zenati M. A new device for beating heart bipolar radiofrequency atrial ablation. $J$ Thorac Cardiovasc Surg. 2003;126:1859-66.

6. Baan J, Van Der Veld E, De Bruin H, Smeenk G, Koops J, Van Dijk A, et al. Continuous measurement of ventricular volume in animals and man by conductance catheter. Circulation. 1984;70:812-23.

7. Packer D, Stevens C, Curley M, Bruce C, Miller F, Khanderia B, et al. Intracardiac phased-array imaging: method and initial clinical experience with high resolution under blood visualization: initial experience with intracardiac phased-array ultrasound. J Am Coll Cardiol. 2002; 39:509-16.

8. Pagel P, Kehl F, Gare M, Hettrick D, Kersten J, Warltier D. Mechanical function of the left atrium: new insights based on analysis of pressure-volume relations and Doppler echocardiography. Anesthesiology. 2003;98:975-94.

9. Jue J, Winslow T, Fazio G, Redberg R, Foster E, Schiller N. Pulsed Doppler characterization of left atrial appendage flow. J Am Soc Echocardiogr. 1993;6:237-44.

10. Blumlein S, Bouchard A, Schiller N, Dae M, Byrd BI, Ports T, et al Quantitation of mitral regurgitation by Doppler echocardiography. Circulation. 1986;74:306-14.

11. Chan S, Kannam J, Douglas P, Manning W. Multiplane transesophageal echocardiographic assessment of left atrial appendage anatomy and function. Am J Cardiol. 1995;76:528-30.

12. Stollberger C, Schneider B, Finsterer J. Elimination of the left atrial appendage to prevent stroke or embolism. Anatomic, physiologic, and pathophysiologic considerations. Chest. 2003;124:2356-62.

13. Melby S, Gaynor S, Lubahn J, Lee A, Rahgozar P, Caruthers S, et al Efficacy and safety of right and left atrial ablations on the beating heart with irrigated bipolar radiofrequency energy: a long-term animal study. J Thorac Cardiovasc Surg. 2006;132:853-60.

14. Davis CA, Rembert JC, Greenfield JC. Compliance of left atrium with and without left atrium appendage. Am J Physiol. 1990;259:H1006-8.

15. Hoit B, Shao Y, Tsai L. Altered left atrial compliance after atrial appendectomy: influence on left atrial and ventricular filling. Circ Res. $1993 ; 72: 167-75$.

16. Tabata T, Oki T, Yamada H. Role of left atrial appendage in left atrial reservoir function as evaluated by left atrial appendage clamping during cardiac surgery. Am J Cardiol. 1998;81:327-32.

17. Massoudy P, Beblo S, Raschke P, Zahler S, Becker B. Influence of intact left atrial appendage on hemodynamic parameters of isolated guinea pig heart. Eur J Med Res. 1998;3:470-4.

18. Kress D, Krum D, Chekanov V, Hare J, Michaud N, Akhtar M, et al Validation of a left atrial lesion pattern for intraoperative ablation of atrial fibrillation. Ann Thorac Surg. 2002;73:1160-8.

19. Sagawa K, Maughan L, Suga H, Sunagawa K. Physiologic Determinations of pressure-volume Relations Cardiac Contraction and the pressure-volume Relationship. New York: Oxford University Press; 1988:110-72.

20. Weiss J, Frederiksen J, Weisfeldt M. Hemodynamic determinants of the time-course of fall in canine left ventricular pressure. J Clin Invest. 1976;58:751-60.

21. Dernellis J, Stefanadis C, Zacharoulis A, Toutouzas P. Left atrial mechanical adaptation to long-standing hemodynamic loads based on pressure-volume relations. Am J Cardiol. 1998;81:1138-43. 